\title{
Comparison of Prospective and Retrospective Methods of a Tigecycline Post-Marketing Surveillance Study in the Safety Outcomes of Patients with Complicated Skin Structure Infection, Complicated Intraabdominal Infection and Community-Acquired Pneumonia
}

Whanhui Chi $\cdot$ Hye Jung Lee $\cdot$ Yong Pil Chong (D)

Received: December 2, 2020 / Accepted: January 6, 2021 / Published online: January 22, 2021

(C) The Author(s) 2021

\begin{abstract}
Introduction: Safety data can be collected through prospective and retrospective methods during post-marketing surveillance (PMS). This study aimed to compare prospective and retrospective methods in terms of examining safety data from PMS of tigecycline.

Methods: This PMS study was an open-label, noncomparative, observational, noninterventional and multicenter study of patients who received tigecycline for infections. From July 2007 to April 2015, 3172 patients were included in this study, of which 738 were enrolled prospectively and 2434 retrospectively. To reduce selection bias, demographic and baseline characteristics were adjusted using 1:2 propensity score matching.

Results: After propensity score matching, data from 1446 patients were analyzed. The incidences of adverse events (AEs) and serious AEs (SAEs) were determined to be significantly
\end{abstract}

Supplementary Information The online version contains supplementary material available at https:// doi.org/10.1007/s40121-021-00398-7.

W. Chi · H. J. Lee

Pfizer Inc, Seoul, Republic of Korea

Y. P. Chong $(\square)$

Department of Infectious Diseases, Asan Medical Center, University of Ulsan College of Medicine, Seoul, Republic of Korea

e-mail: drchong@amc.seoul.kr higher in the prospective method compared with those of the retrospective method $(P<0.001$ and $P=0.004$, respectively). However, no significant differences in the incidences of adverse drug reactions (ADRs) and serious ADRs (SADRs) were detected between the two groups ( $P=0.09$ and $P=0.33$, respectively). In a subgroup analysis of 360 patients from 14 hospitals involved in both prospective and retrospective methods, the incidence of AEs was found to be significantly higher using the prospective method compared with when the retrospective method was used $(P<0.001)$, but there were no significant differences in ADRs $(P=0.14)$, SAEs $(P=0.24)$ and SADRs.

Conclusion: In general, the prospective method can detect safety data effectively in a PMS study, whereas retrospective data collection may be an alternative option in collecting ADR data when a prospective PMS study is not deemed feasible.

Keywords: Community-acquired pneumonia; Complicated intraabdominal infection; Complicated skin structure infection; Postmarketing surveillance; Prospective study; Retrospective study; Safety; Tigecycline 


\section{Key Summary Points}

This study was unique in that the safety data from both prospective and retrospective methods were collected for comparison in a single post-marketing surveillance (PMS) study

Propensity score matching was used to reduce selection bias and to adjust confounding variables between the prospective and retrospective methods. Overall, the prospective method detected safety outcomes well. Adverse events and serious adverse events were more frequently reported in the prospective data collection method

The incidence of adverse drug reactions (ADRs) was similar between the two methods, especially in patients treated with tigecycline for $>14$ days

A retrospective PMS study may be used as an alternative for detecting ADRs, especially for the antimicrobial agent administered for several weeks, when various limitations prevent prospective data collection

\section{DIGITAL FEATURES}

This article is published with digital features, including a summary slide, to facilitate understanding of the article. To view digital features for this article go to https://doi.org/10.6084/ m9.figshare.13525796.

\section{INTRODUCTION}

The re-examination of drugs, including new ones for post-marketing safety management, has been required in many countries, including South Korea. The re-examination systems aim to determine the occurrence of adverse events (AEs), including AEs that did not appear in premarketing clinical trials for the period set by the health authority (e.g., 4 or 6 years), and to monitor both common and rare AEs and adverse drug reactions (ADRs) [1-4].

Tigecycline is a first-in-class glycylcycline antibacterial drug approved for the treatment of complicated skin structure infections (cSSTI), complicated intraabdominal infections (cIAI) and community-acquired bacterial pneumonia (CAP) in patients 18 years of age and older. Tigecycline was approved in July 2007 in South Korea. As per the South Korea's Ministry of Food and Drug Safety requirements, safety and efficacy information for new drugs should be prospectively collected in at least 3000 patients who receive the drug in routine clinical practice within the 6 years after its approval (until July 2013 for tigecycline) [2]. South Korea has a national health insurance system with universal coverage. In most cases, tigecycline is administered to severely ill patients as it is reimbursed only as a second-line therapy. Thus, enrollment of patients for the prospective post-marketing surveillance (PMS) was difficult, leading to an insufficient number of patients during the original prospective re-examination period. Accordingly, the Ministry of Food and Drug Safety permitted an additional 1 year in order to enroll a sufficient number of patients through the retrospective method.

Tigecycline PMS was the first PMS study conducted in South Korea through both prospective and retrospective methods, and this PMS study has the most tigecycline real-world data in Asia. No other PMS studies compare the results of prospective and retrospective safety data collections for an antimicrobial agent. Thus, the purpose of this study is to compare the safety data of tigecycline gathered via prospective and retrospective methods. The collected safety data included AEs, serious AEs (SAEs), ADRs and serious ADRs (SADRs).

\section{METHODS}

\section{Data Source}

Tigecycline PMS was an open-label, noncomparative, observational, noninterventional and multicenter study, examining the safety of tigecycline therapy for patients receiving it. 
Safety data were collected in two periods using prospective and retrospective methods. In the prospective method, 27 hospitals were involved, but the target sample size of 3000 patients was not achieved. Additional safety data were then collected in the following period using the retrospective method wherein 17 hospitals were involved. Patients who received at least one dose of tigecycline for any of the approved indications on the product label were eligible for the study.

Period I (July 2007 to July 2013) involved prospective collection of data. Patients receiving tigecycline according to the product label, who agreed to sign a data privacy statement, were enrolled in this study. Observations were conducted from the initial administration of tigecycline until the end of treatment or test of cure, which was performed within 14 days after the last tigecycline administration. Comorbidities and the use of concomitant medications were recorded in case report forms. All abnormal findings including AEs during tigecycline therapy were assessed and documented by physicians during the observation period. For the retrospective data collection in period II (April 2014 to April 2015), relevant data were collected by reviewing medical records of patients who had received at least one dose of tigecycline. Similar to the prospective phase, data were collected from the start of tigecycline therapy until the end of treatment or test of cure. The eligibility criteria were the same as those of the prospective method. Detailed information about all concomitant medications and comorbidities was also recorded.

Data from 3172 patients, including those prospectively collected from 738 and those retrospectively collected from 2434 , were included in this study. Seven patients with lacking data on the demographic and baseline characteristics were excluded. Finally, 3165 patients were included in the safety evaluation population.

This was a surveillance study mandated by the Ministry of Food and Drug Safety to collect information from patients receiving the drug. The Institutional Review Board of each participating center approved this study according to institutional rules (Supplementary Table S4). In the prospective study, informed consent was obtained from each participant.

\section{Variables}

\section{Safety End Point}

Safety end points included the incidence of AEs, ADRs, SAEs and SADRs that occurred after tigecycline administration during the observation period. AE was defined as an adverse outcome that occurred while a patient was receiving tigecycline, but the event was not necessarily caused by tigecycline. Meanwhile, ADR was defined as a harmful or unpleasant reaction that had a causal relationship with tigecycline. SAE was defined as any AE that resulted in death, was life-threatening (immediate risk of death), required hospitalization (or prolonged hospitalization), resulted in persistent or significant disability/incapacity (substantial disruption of the ability to conduct normal life functions), resulted in congenital anomaly/birth defect or was an important medical event that had jeopardized the patient or required intervention to prevent one of the other AEs listed in the definition above [5]. Lastly, SADR was defined as any ADR with the same definition as above.

\section{Demographic and Baseline Characteristics}

A physician collected the demographic characteristics and other medical conditions at baseline and further recorded the information in each patient's case report form. Demographic characteristics included sex and age. Medical conditions at baseline included infection site, severity of infection, underlying disease/condition, kidney disorders, liver disorders and concomitant medications. The total administration period of tigecycline was also recorded.

\section{Statistical Analysis}

\section{Adjustments of Covariate}

If demographic and/or baseline characteristics varied depending on the data collection method, the safety end points may be affected by these variables. To correct bias associated with the data collection method, the effects of demographic characteristics and medical 
Table 1 Assessment of balance of the distributions of demographic and baseline characteristics before and after propensity score matching

\begin{tabular}{|c|c|c|c|c|c|c|}
\hline \multirow[t]{2}{*}{ Variable } & \multicolumn{2}{|l|}{ All patients } & \multirow[t]{2}{*}{$p$ value } & \multicolumn{2}{|c|}{ Propensity-matched patients } & \multirow[t]{2}{*}{$P$ value } \\
\hline & $\begin{array}{l}\text { Prospective } \\
(N=734)\end{array}$ & $\begin{array}{l}\text { Retrospective } \\
(N=2431)\end{array}$ & & $\begin{array}{l}\text { Prospective } \\
(\boldsymbol{N}=482)\end{array}$ & $\begin{array}{l}\text { Retrospective } \\
(N=964)\end{array}$ & \\
\hline \multicolumn{7}{|l|}{$\overline{S e x}$} \\
\hline Male & $488(66.5)$ & $1589(65.4)$ & 0.58 & $312(64.7)$ & $616(63.9)$ & 0.76 \\
\hline Female & $246(33.5)$ & $842(34.6)$ & & $170(35.3)$ & $348(36.1)$ & \\
\hline \multicolumn{7}{|l|}{ Age (years) } \\
\hline$<30$ & $28(3.8)$ & $98(4.0)$ & 0.33 & $12(2.5)$ & $18(1.9)$ & 0.92 \\
\hline $30-39$ & $37(5.0)$ & $163(6.7)$ & & $21(4.4)$ & $40(4.2)$ & \\
\hline $40-49$ & $79(10.8)$ & $267(11.0)$ & & $48(10.0)$ & $90(9.3)$ & \\
\hline $50-64$ & $250(34.1)$ & $865(35.6)$ & & $176(36.5)$ & $366(38.0)$ & \\
\hline$\geq 65$ & $340(46.3)$ & $1038(42.7)$ & & $225(46.7)$ & $450(46.7)$ & \\
\hline \multicolumn{7}{|c|}{ Infection site } \\
\hline cSSTI & $283(38.6)$ & $693(28.5)$ & $<0.001$ & $201(41.7)$ & $391(40.6)$ & 0.91 \\
\hline CAP & $61(8.3)$ & $181(7.5)$ & & $10(2.1)$ & $21(2.2)$ & \\
\hline cIAI & $390(53.1)$ & $1557(64.1)$ & & $271(56.2)$ & $552(57.3)$ & \\
\hline \multicolumn{7}{|c|}{ Severity of infection } \\
\hline Mild & $71(9.7)$ & $216(8.9)$ & $<0.001$ & $17(3.5)$ & $35(3.6)$ & 0.96 \\
\hline Moderate & $532(72.5)$ & $1,537(63.2)$ & & $369(76.6)$ & $743(77.1)$ & \\
\hline Severe & $131(17.9)$ & $678(27.9)$ & & $96(19.9)$ & $186(19.3)$ & \\
\hline \multicolumn{7}{|c|}{ Underlying disease/condition } \\
\hline Yes & $532(72.5)$ & $2380(97.9)$ & $<0.001$ & $482(100)$ & $964(100)$ & - \\
\hline No & $202(27.5)$ & $51(2.1)$ & & 0 & 0 & \\
\hline \multicolumn{7}{|c|}{ Kidney disorder } \\
\hline Yes & $119(16.2)$ & $486(20.0)$ & 0.02 & $111(23.0)$ & $210(21.8)$ & 0.59 \\
\hline No & $615(83.8)$ & $1945(80.0)$ & & $371(77.0)$ & $754(78.2)$ & \\
\hline \multicolumn{7}{|c|}{ Liver disorder } \\
\hline Yes & $138(18.8)$ & $776(31.9)$ & $<0.001$ & $133(27.6)$ & $253(26.2)$ & 0.58 \\
\hline No & $596(81.2)$ & $1655(68.1)$ & & $349(72.4)$ & $711(73.8)$ & \\
\hline \multicolumn{7}{|c|}{ Concomitant medication } \\
\hline Yes & $630(85.8)$ & 2407 (99) & $<0.001$ & $482(100)$ & $964(100)$ & - \\
\hline No & $104(14.2)$ & $24(1.0)$ & & 0 & 0 & \\
\hline Total durati & on of tigecycl & apy (days) & & & & \\
\hline
\end{tabular}


Table 1 continued

\begin{tabular}{|c|c|c|c|c|c|c|}
\hline \multirow[t]{2}{*}{ Variable } & \multicolumn{2}{|l|}{ All patients } & \multirow[t]{2}{*}{$p$ value } & \multicolumn{2}{|c|}{ Propensity-matched patients } & \multirow[t]{2}{*}{$P$ value } \\
\hline & $\begin{array}{l}\text { Prospective } \\
(N=734)\end{array}$ & $\begin{array}{l}\text { Retrospective } \\
(N=2431)\end{array}$ & & $\begin{array}{l}\text { Prospective } \\
(N=482)\end{array}$ & $\begin{array}{l}\text { Retrospective } \\
(N=964)\end{array}$ & \\
\hline$\leq 14$ & $453(61.7)$ & $1566(64.4)$ & 0.18 & $297(61.6)$ & $581(60.3)$ & 0.62 \\
\hline$>14$ & $281(38.3)$ & $865(35.6)$ & & $185(38.4)$ & $383(39.7)$ & \\
\hline
\end{tabular}

Data are presented as the number of patients (with the corresponding percentage shown in parentheses) cSSTI complicated skin structure infection, CAP community-acquired pneumonia, cIAI complicated intraabdominal infection

Table 2 Comparison of safety outcomes between the prospective and retrospective methods

\begin{tabular}{|c|c|c|c|c|c|c|}
\hline \multirow[t]{2}{*}{ Safety outcome } & \multicolumn{2}{|l|}{ All patients } & \multirow[t]{2}{*}{$p$ value } & \multicolumn{2}{|c|}{ Propensity-matched patients } & \multirow[t]{2}{*}{$P$ value } \\
\hline & $\begin{array}{l}\text { Prospective } \\
(N=734)\end{array}$ & $\begin{array}{l}\text { Retrospective } \\
(N=2431)\end{array}$ & & $\begin{array}{l}\text { Prospective } \\
(N=482)\end{array}$ & $\begin{array}{l}\text { Retrospective } \\
(N=964)\end{array}$ & \\
\hline Adverse event (AE) & $250(34.1)$ & $793(32.6)$ & 0.47 & $219(45.4)$ & $304(31.5)$ & $<0.001$ \\
\hline $\begin{array}{l}\text { Adverse drug } \\
\text { reaction (ADR) }\end{array}$ & $84(11.4)$ & $228(9.4)$ & 0.10 & $68(14.1)$ & $106(11.0)$ & 0.09 \\
\hline Serious AE & $79(10.8)$ & $340(14.0)$ & 0.02 & $75(15.6)$ & $99(10.3)$ & 0.004 \\
\hline Serious ADR & $1(0.1)$ & $4(0.2)$ & $>0.99$ & $1(0.2)$ & 0 & 0.33 \\
\hline
\end{tabular}

Data are presented as the number of patients (with the corresponding percentage shown in parentheses)

conditions at baseline were adjusted using propensity score matching before conducting the safety analysis. Propensity score matching was performed in four steps. First, we selected covariates for the propensity score calculation in patients with underlying diseases/conditions and concurrent medication. Covariates were as follows: sex, age, infection sites, severity of infection, kidney disorders and liver disorders. Second, a logistic regression model was utilized with the selected covariate variables in calculating the propensity score for each patient. Third, the nearest neighbor method was used to match the propensity scores; the ratio of prospective and retrospective methods was 1:2. Finally, significant differences in covariate variables between data collection methods were determined using chi-square and Fisher's exact tests to examine covariate balance before and after matching. As part of the exploratory analysis, additional analyses were conducted using the data from 14 hospitals that were involved in both prospective and retrospective data collections.

\section{Safety Analyses}

The incidences of AEs, ADRs, SAEs and SADRs for the two methods of data collection were compared using chi-square and Fisher's exact tests at a statistically significant level of 5\%. All statistical analyses were performed using the SAS package version 9.4 (SAS Institute Inc., Cary, NC) or R version 3.3.0 programs.

\section{RESULTS}

No significant differences in sex, age and the total duration of tigecycline therapy were noted 
Table 3 Demographic and baseline characteristics of patients from 14 hospitals that participated in both prospective and retrospective studies

\begin{tabular}{|c|c|c|c|c|c|c|}
\hline \multirow[t]{2}{*}{ Variable } & \multicolumn{2}{|l|}{ All patients } & \multirow[t]{2}{*}{$p$ value } & \multicolumn{2}{|c|}{ Propensity-matched patients } & \multirow[t]{2}{*}{$P$ value } \\
\hline & $\begin{array}{l}\text { Prospective } \\
(N=141)\end{array}$ & $\begin{array}{l}\text { Retrospective } \\
(N=2256)\end{array}$ & & $\begin{array}{l}\text { Prospective } \\
(N=120)\end{array}$ & $\begin{array}{l}\text { Retrospective } \\
(N=240)\end{array}$ & \\
\hline \multicolumn{7}{|l|}{$\overline{\text { Sex }}$} \\
\hline Male & $103(73.1)$ & $1471(65.2)$ & 0.06 & $85(70.8)$ & $164(68.3)$ & 0.63 \\
\hline Female & $38(27.0)$ & $785(34.8)$ & & $35(29.2)$ & $76(31.7)$ & \\
\hline \multicolumn{7}{|l|}{ Age (years) } \\
\hline$<30$ & $6(4.3)$ & $95(4.2)$ & 0.95 & $4(3.3)$ & $5(2.1)$ & 0.96 \\
\hline $30-39$ & $11(7.8)$ & $155(6.9)$ & & $8(6.7)$ & $17(7.1)$ & \\
\hline $40-49$ & $15(10.6)$ & $253(11.2)$ & & $10(8.3)$ & $20(8.3)$ & \\
\hline $50-64$ & $54(38.3)$ & $808(35.8)$ & & $50(41.7)$ & $104(43.3)$ & \\
\hline$\geq 65$ & $55(39.0)$ & $945(41.9)$ & & $48(40.0)$ & $94(39.2)$ & \\
\hline \multicolumn{7}{|c|}{ Infection site } \\
\hline cSSTI & $62(44.0)$ & $668(29.6)$ & $<0.001$ & $49(40.8)$ & $99(41.3)$ & $>0.99$ \\
\hline CAP & $3(2.1)$ & $161(7.1)$ & & $3(2.5)$ & $6(2.5)$ & \\
\hline cIAI & $76(53.9)$ & $1427(63.3)$ & & $68(56.7)$ & $135(56.3)$ & \\
\hline \multicolumn{7}{|c|}{ Severity of infection } \\
\hline Mild & $8(5.7)$ & $189(8.4)$ & 0.22 & $6(5.0)$ & $10(4.2)$ & 0.90 \\
\hline Moderate & $87(61.7)$ & $1462(64.8)$ & & $76(63.3)$ & $157(65.4)$ & \\
\hline Severe & $46(32.6)$ & $605(26.8)$ & & $38(31.7)$ & $73(30.4)$ & \\
\hline \multicolumn{7}{|c|}{ Underlying disease/condition } \\
\hline Yes & $126(89.4)$ & $2207(97.8)$ & $<0.001$ & $120(100.0)$ & $240(100)$ & - \\
\hline No & $15(10.6)$ & $49(2.2)$ & & 0 & 0 & \\
\hline \multicolumn{7}{|c|}{ Kidney disorder } \\
\hline Yes & $30(21.3)$ & $459(20.4)$ & 0.79 & $29(24.2)$ & $54(22.5)$ & 0.72 \\
\hline No & $111(78.7)$ & $1797(79.7)$ & & $91(75.8)$ & $186(77.5)$ & \\
\hline \multicolumn{7}{|l|}{$\begin{array}{l}\text { Liver } \\
\text { disorder }\end{array}$} \\
\hline Yes & $31(22.0)$ & $711(31.5)$ & 0.02 & $31(25.8)$ & $61(25.4)$ & 0.93 \\
\hline No & $110(78.0)$ & $1545(68.5)$ & & $89(74.2)$ & $179(74.6)$ & \\
\hline \multicolumn{7}{|c|}{ Concomitant medications } \\
\hline Yes & $135(95.7)$ & $2232(98.9)$ & 0.01 & $120(100)$ & $240(100)$ & - \\
\hline No & $6(4.3)$ & $24(1.1)$ & & 0 & 0 & \\
\hline \multicolumn{7}{|c|}{ Total duration of tigecycline therapy (days) } \\
\hline
\end{tabular}


Table 3 continued

\begin{tabular}{lllllll}
\hline Variable & All patients & & $\boldsymbol{p}$ value & \multicolumn{2}{l}{ Propensity-matched patients } & \multirow{2}{P}{ value } \\
\cline { 2 - 3 } \cline { 5 - 6 } & $\begin{array}{l}\text { Prospective } \\
(\boldsymbol{N}=\mathbf{1 4 1})\end{array}$ & $\begin{array}{l}\text { Retrospective } \\
(\boldsymbol{N}=\mathbf{2 2 5 6})\end{array}$ & & $\begin{array}{l}\text { Prospective } \\
(\boldsymbol{N}=\mathbf{1 2 0})\end{array}$ & $\begin{array}{l}\text { Retrospective } \\
(\boldsymbol{N}=\mathbf{2 4 0})\end{array}$ & \\
\hline$\leq 14$ & $77(54.6)$ & $1455(64.5)$ & 0.02 & $65(54.2)$ & $144(60.0)$ & 0.29 \\
$>14$ & $64(45.4)$ & $801(35.5)$ & & $55(45.8)$ & $96(40.0)$ & \\
\hline
\end{tabular}

Data are presented as the number of patients (with the corresponding percentage shown in parentheses) cSSTI complicated skin structure infection, CAP community-acquired pneumonia, cIAI complicated intraabdominal infection

Table 4 Comparison of safety outcomes between the prospective and retrospective methods in propensity-matched patients from 14 hospitals that participated in both prospective and retrospective studies

\begin{tabular}{|c|c|c|c|}
\hline \multirow[t]{2}{*}{ Safety outcome } & \multicolumn{2}{|c|}{ Propensity-matched patients from 14 hospitals } & \multirow[t]{2}{*}{$P$ value } \\
\hline & Prospective $(N=120)$ & Retrospective $(N=240)$ & \\
\hline Adverse event (AE) & $64(53.3)$ & $71(29.6)$ & $<0.001$ \\
\hline Adverse drug reaction (ADR) & $14(11.7)$ & $17(7.1)$ & 0.14 \\
\hline Serious AE & $13(10.8)$ & $37(15.4)$ & 0.24 \\
\hline Serious ADR & 0 & 0 & - \\
\hline
\end{tabular}

Data are presented as the number of patients (with the corresponding percentage shown in parentheses)

between the prospective and retrospective methods (Table 1). However, patients in the retrospective method group were significantly more likely to have cIAI, severe infections, underlying diseases/conditions, kidney disorders, liver disorders and concomitant medications compared to those in the prospective method group. Therefore, propensity score matching was performed to adjust for differences in demographic characteristics and medical conditions at baseline. As a result, 482 patients in the prospective method group and 964 in the retrospective method group were selected. The differences between the two groups were corrected after propensity score matching, as shown in Table 1.

Safety outcomes for tigecycline are provided in Table 2. AEs, ADRs and SADRs were determined to be similar in the two groups. SAEs occurred more frequently in the retrospective group than in the prospective group, reflecting the severe underlying condition of the former group $(P=0.02)$. However, in the propensityadjusted analysis, AEs and SAEs were more frequently reported in the prospective group than in the retrospective group $(P<0.001$ and $P=0.004$, respectively). The incidences of ADRs were not significantly different between the two groups, and the types and proportions of ADRs were similar (Supplementary Table S1). When the propensity-matched cohort was divided into two subgroups according to the duration of tigecycline therapy ( $\leq 14$ days vs. $>14$ days), the demographic and baseline characteristics were generally similar between the prospective and retrospective groups regardless of the duration of therapy. (Supplementary Table S2). In patients treated for $\leq 14$ days, AEs, ADRs and SAEs were significantly more frequently reported in the prospective group than in the retrospective group $(P<0.001, \quad P=0.01$ and $P=0.002, \quad$ respectively) (Supplementary Table S3). However, in patients treated for $>14$ days, the incidences of ADRs and SAEs 
were similar between the two groups $(P=0.85$ and $P=0.66$, respectively).

To decrease the inter-investigator differences, we compared the data collected from 14 hospitals involved in both the prospective and retrospective data collections. We analyzed an additional 2397 patients, including 141 from the prospective method and 2256 from the retrospective method. In total, 120 patients in the prospective method and 240 patients in the retrospective method were selected by propensity score matching. The demographic and baseline characteristics were found to be comparable between the two groups after propensity score matching (Table 3). The safety outcomes for tigecycline in these patients are shown in Table 4. As per our findings, AEs were more frequently reported in the prospective group than in the retrospective group $(P<0.001)$. However, the incidences of ADRs and SAEs were not significantly different between the two groups.

\section{DISCUSSION}

Safety outcomes for tigecycline from prospectively collected data were compared to retrospectively collected PMS data in patients with cSSTI, cIAI and CAP during routine clinical practice in South Korea. We were able to reduce selection bias and adjusted confounding variables using propensity score matching as patients in the retrospective group had more underlying diseases/conditions and were more severely ill than those in the prospective group. AEs and SAEs were more frequently detected using the prospective method unlike when the retrospective method was used, but the incidences of ADRs and SADRs were not statistically different between the two data collection methods. These results indicate that the retrospective method detects AEs less than the prospective method in real clinical practice; however, ADRs were detected at similar rates using both methods. This reflects real clinical practice in which ADRs are rarely omitted from the medical record.

In a PMS study of tigecycline, treatmentemergent AEs were determined to occur in
$60.4 \%$ of patients regardless of study drug causality. The most common treatment-emergent AEs in this pivotal study were nausea, vomiting and diarrhea $[6,7]$. These common AEs were similarly detected in our PMS study, although the incidence of AEs was slightly lower compared with previous PMS studies. In a retrospective study of elderly patients with multidrug-resistant bacterial infections, $42.6 \%$ of these patients experienced AEs associated with the use of tigecycline; these AEs include gastrointestinal symptoms, such as nausea and vomiting [8]. In another retrospective study of tigecycline in the treatment of ventilator-associated pneumonia, $26 \%$ of the patients experienced side effects, including liver damage and gastrointestinal symptoms [9]. In our PMS study, $32.6 \%$ of patients from the retrospective data collection group experienced AEs, such as nausea and vomiting. Thus, our data are consistent with other studies confirming proper data collection.

In our study, 14 hospitals participated in both prospective and retrospective studies. In the subgroup analysis of data from these hospitals, data were analyzed through propensity score matching to decrease inter-investigator variability. More AEs were detected through the prospective method $(P<0.001)$. However, no significant differences were noted in the incidences of ADRs and SAEs between the prospective and retrospective methods. These results indicate that severe safety outcome and drugrelated events are appropriately detected by both methods.

Moreover, the prospective method has been determined to be more valuable in terms of collecting safety information since data collection can be controlled and monitored and variables can be measured consistently [10]. Meanwhile, retrospective data collection is relatively inexpensive, quick and easy to perform, and more patients can be recruited [10-12]. Our findings suggest that a retrospective PMS study may be used as an alternative for detecting ADRs and SAEs, especially for the drug administered for several weeks, when various limitations prevent the prospective collection of data or when safety data from a large number of patients are needed for rare diseases. 
Our study has several limitations. First, the number of enrolled patients was quite different between the prospective and retrospective methods. Second, a limited number of hospitals participated in both data collection methods. Although propensity score matching and site matching were used to overcome these limitations, further studies should be conducted to generalize our findings. Despite these limitations, this analysis is deemed meaningful. The safety information was collected in a single study through both prospective and retrospective methods. Furthermore, to the best of our knowledge, this is the first analysis to compare prospective and retrospective methods in terms of collecting PMS safety data for an antimicrobial agent.

\section{CONCLUSION}

A prospective data collection method can detect safety data more precisely than a retrospective method for PMS studies. However, retrospective data collection may be a good alternative option for collecting ADR data when a prospective PMS study is not deemed feasible.

\section{ACKNOWLEDGEMENTS}

We thank the tigecycline PMS investigators for their valuable involvement in this study: YoonSoo Park (Gachon University Gil Medical Center), Jacob Lee (Kangnam Sacred Heart Hospital), Jun Seong Son (Kyung Hee University Hospital at Gangdong), Joong Sik Eom (Kangdong Sacred Heart Hospital), Joon-Sup Yeom (Kangbuk Samsung Hospital), Shin-Woo Kim (Kyungpook National University Hospital), Mi Suk Lee (Kyung Hee University Medical Center), Seong Yeol Ryu (Keimyung University Dongsan Medical Center), Hyun Ah Kim (Keimyung University Dongsan Medical Center), Won Suk Choi (Korea University Ansan Hospital), Jang Wook Sohn (Korea University Anam Hospital), Ji-Young Park (Kosin University Gospel Hospital), Ki-Deok Lee (Nowon Eulji Medical Center), Ki Tae Kwon (Daegu Fatima Hospital), HyunHee Kwon (Daegu Catholic University Medical
Center), Dong Sik Jung (Dong-A University Hospital), Cheol In Kang (Samsung Medical Center), Sang Hoon Han (Severance Hospital), Kyoung Won Jung (Ajou University Hospital), Jian Hur (Yeungnam University Medical Center), Jae-Bum Jun (Ulsan University Hospital), Jae Hoon Lee (Wonkwang University Hospital), Young Keun Kim (Wonju Severance Christian Hospital), Jin-Soo Lee (Inha University Hospital), Chang-Seop Lee (Jeonbuk National University Hospital), Sang Taek Heo (Jeju National University Hospital), Yun Tae Chae (Hanil General Hospital).

Funding. Pfizer Pharmaceuticals Korea Ltd. sponsored this research. The study sponsor also funded the journal's Rapid Service Fee.

Medical writing and editorial assistance. We thank a medical writer and English editors for medical writing and editorial assistance: Hyun-Ji Kim from MediHelpLine Co., Ltd., and Crimson Interactive Korea Co., Ltd.

Authorship. All named authors meet the International Committee of Medical Journal Editors (ICMJE) criteria for authorship for this article, take responsibility for the integrity of the work as a whole, and have given their approval for this version to be published.

Authorship Contributions. All authors participated in the design or implementation or analysis, and interpretation of the study and in the development of this manuscript. All authors had full access to the data and gave final approval before submission. The corresponding author was responsible for the publication submission.

Disclosures. Yong Pil Chong has received a research grant from Pfizer Pharmaceuticals Korea Ltd. Whan Hui Chi and Hye Jung Lee are full-time employees of Pfizer Pharmaceuticals Korea Ltd. Hye Jung Lee has changed her affiliation after the completion of the manuscript from Pfizer Korea to Viatris Korea.

Compliance with Ethics Guidelines. This was a surveillance study mandated by the 
Ministry of Food and Drug Safety to collect information from patients receiving the drug. The Institutional Review Board of each participating center approved this study according to institutional rules (Supplementary Table S4). In the prospective study, informed consent was obtained from each participant.

Data Availability. The datasets generated and/or analysed during the current study are not publicly available as this is outside the terms of the ethics approval.

Open Access. This article is licensed under a Creative Commons Attribution-NonCommercial 4.0 International License, which permits any non-commercial use, sharing, adaptation, distribution and reproduction in any medium or format, as long as you give appropriate credit to the original author(s) and the source, provide a link to the Creative Commons licence, and indicate if changes were made. The images or other third party material in this article are included in the article's Creative Commons licence, unless indicated otherwise in a credit line to the material. If material is not included in the article's Creative Commons licence and your intended use is not permitted by statutory regulation or exceeds the permitted use, you will need to obtain permission directly from the copyright holder. To view a copy of this licence, visit http://creativecommons.org/licenses/bync/4.0/.

\section{REFERENCES}

1. Park EJ. The quality control of post-authorization study of re-examination in Korea. J Korean Acad Manag Care Pharm. 2015;4(1):1-6.

2. KFDA. Guideline for re-examination on drugs including new drugs. Cheongju: KFDA; 2012.
3. Suvarna V. Phase IV of drug development. Perspect Clin Res. 2010;1(2):57-60.

4. Faich GA. Postmarketing surveillance: lessons learned. Drug Inf J. 1991;25(4):531-5. https://doi. org/10.1177/009286159102500409.

5. U.S. Food and Drug Administration. What is a Serious Adverse Event? 2020. https://www.fda.gov/ safety/reporting-serious-problems-fda/whatserious-adverse-event. Accessed 9 Nov 2020.

6. Fomin P, Beuran M, Gradauskas A, Barauskas G, Datsenko A, Dartois N, Ellis-Grosse E, Loh E. Tigecycline is efficacious in the treatment of complicated intra-abdominal infections. Int $\mathrm{J}$ Surg. 2005;3(1):35-47.

7. Oliva ME, Rekha A, Yellin A, Pasternak J, Campos M, Rose GM, Babinchak T, Ellis-Grosse EJ, Loh E, the 301 Study Group. A multicenter trial of the efficacy and safety of tigecycline versus imipenem/cilastatin in patients with complicated intra-abdominal infections [Study ID Numbers: 3074A1-301-WW; ClinicalTrials. gov Identifier: NCT00081744]. BioMed Central Infect Dis. 2005; $51: 88$.

8. Xia G, Jiang R. Clinical study on the safety and efficacy of high-dose tigecycline in the elderly patients with multidrug-resistant bacterial infections: a retrospective analysis. Medicine (Baltimore). 2020;99(10):e19466. https://doi.org/10. 1097/MD.0000000000019466.

9. Chen Z, Shi X. Adverse events of high-dose tigecycline in the treatment of ventilator-associated pneumonia due to multidrug-resistant pathogens. Medicine (Baltimore). 2018;97(38):e12467. https:// doi.org/10.1097/MD.0000000000012467.

10. Ukai T, Shikata S, Nakayama T, Takemura YC. A comparison of the results of prospective and retrospective cohort studies in the field of digestive surgery. Jpn J Surg. 2017;47(7):789-94.

11. Sedgwick P. Retrospective cohort studies: advantages and disadvantages. BMJ. 2014;348:g1072.

12. Weinger M, Slagle J, Ordonez N. Retrospective data collection and analytical techniques for patient safety studies. J Biomed Inform. 2003;36(1-2): 106-19. 